\title{
Early-age creep behaviour of 3D printable mortars: Experimental characterisation and analytical modelling
}

\author{
Laura Esposito (1D - Lorenzo Casagrande - Costantino Menna • Domenico Asprone • \\ Ferdinando Auricchio
}

Received: 21 May 2021/Accepted: 29 September 2021 / Published online: 29 October 2021

(C) The Author(s) 2021

\begin{abstract}
The construction sector is experiencing significant technological innovations with digitalisation tools and automated construction techniques, such as additive manufacturing. Additive manufacturing utilising cement-based materials can potentially remove the technological/economic barriers associated with innovative architectural/structural shapes which are not suitable for conventional formworks adopted for concrete material. However, in the "freeform" digital fabrication with concrete, the mechanical properties prediction of the material in the fresh state is essential for controlling both the element deformations and overall stability during printing. In this paper, the authors explore the critical aspects related to the determination of the early-age creep properties of a 3D printable cement-based material, particularly investigating such a behaviour at different resting times. The experimental results are used to calibrate the Burgers' analytical model to consider both the elastic and the viscous response of the $3 \mathrm{D}$ printable mortar investigated in the fresh state. The visco-elastic model is validated by comparing the
\end{abstract}

L. Esposito $(\bowtie) \cdot$ C. Menna · D. Asprone

Department of Structures for Engineering and

Architecture, University of Naples Federico II, Via

Claudio 21, 80125 Naples, Italy

e-mail: laura.esposito2@unina.it

L. Casagrande · F. Auricchio

Department of Civil Engineering and Architecture,

University of Pavia, Via Ferrata 3, 27100 Pavia, Italy analytical total strain vs time curve with the corresponding experimental counterpart replicating the layer-by-layer stacking process in the 3D concrete printing process. It was found that the Burgers' model represents a valuable numerical approach to evaluate the overall accumulation of layer deformation of a 3D printed element, since it is capable of taking into account the time dependency due to the time gap and the variable material stiffness over the process time.

Keywords Early-age creep · 3D concrete printing . Burgers' model $\cdot$ Compressive tests

\section{Introduction}

The 3D concrete printing (3DCP) $[1,2]$ technique consists of extruding and then depositing fresh concrete filaments without formworks that are generally adopted to confine and stabilise the poured material in the traditional formative processes. The 3DCP technique starts with the pumping of the cementitious mortar, whose composition is designed to provide a thixotropic behaviour: the shear-thinning property of thixotropic materials implies that the viscosity and the yield stress decrease if external energy is applied. Furthermore, once the material comes out from the nozzle, i.e., when the external energy is removed, the yield stress grows again in a specific time interval due to the re-flocculation [3], and the buildability 
requirement can be satisfied. Indeed, the fresh concrete mix should ensure adequate yield stress, stiffness, and stability to sustain its self-weight and the weight of the filaments above it; thus, the printable cementitious mortar must satisfy specific rheological and mechanical requirements resulting into specific buildability properties (just after the extrusion) $[4,5]$. An additional requirement is the shape retention of the extruded filament that ensures the printed object to maintain its shape during the printing process; consistently, this requirement also relies on the printing velocity and geometry of the nozzle [6]. Nevertheless, the prediction of the actual behaviour of such innovative printable materials is not straightforward, especially in terms of deformations during printing; such an issue is associated with the technological novelties of digital fabrication with concrete and there is limited knowledge in this field.

The 3D printable concrete in the fresh state (i.e., from pumping to layer deposition) behaves predominantly as a visco-elastic material [7-9]. Generally, viscoelasticity combines viscous and elastic behaviours; the key properties of visco-elastic materials are the stress relaxation (i.e., the stress level decreases under constant applied strain over time) and the creep effect (i.e., the strain level increases under constant applied load over time). In order to distinguish and define the limit between pure viscous and elastic behaviours, the dimensionless Deborah (De) number is employed in rheology and it is defined as the ratio of two characteristic times [10]: the relaxation time $t_{r}$ [sec] (i.e., time for the material to reach the equilibrium after perturbation) and the observation time $T$ [sec]. A low value of the De number means that the material behaves as a viscous Newtonian fluid; on the contrary, if the De value is high, the material behaviour is dominated by elasticity. Since fresh cementitious materials initiate the hardening after the extrusion from the nozzle, a transition from a viscoelastic to an elastic behaviour is expected: such transition is driven by flocculation mechanisms and cement hydration kinetics over time and depends on the specific material utilised in the 3DCP process. The layer-by-layer deposition occurring during 3DCP generates progressive compression in the concrete filaments, which undergo increasing levels of axial strain while the hardening process takes place simultaneously. Additionally, delayed deformations may also appear during the automated process, affecting either the dimensional accuracy (i.e., through the accumulation of layer deformation) or the buildability/ stability performance of the printed element. Hence, at a very early-age, it is fundamental to characterise the long-term compressive response of printable mortars as it can lead to additional (and/or not negligible) deformations under the progressively applied load. The mix design of printable mortars is generally characterised by a high paste volume, a low water-tocement ratio $(w / c)$, a high dosage of mineral additions and superplasticiser, often mixed with a viscositymodifying agent [11]. Such particularities affect the visco-elastic properties of the resulting fresh material: on the one hand, low water-to-cement ratios yield higher compressive strengths over time; on the other hand, high paste volumes are more sensitive to creep and shrinkage [12-14]. The evolution over time of the degree of hydration strongly influences the mechanical response in cement-based materials; therefore, higher creep strains are experienced if the traditional concrete is demoulded and loaded at early ages than that in the hardened state $[12,15-20]$. It is reasonable to expect a similar behaviour in the cementitious materials employed in the 3DCP.

However, one of the main challenges for the earlyage characterisation of printable mortars is to control the evolution over time of the visco-elastic material properties during the test execution. In order to overcome this issue, it is important to define specific testing procedures, especially in terms of test duration. Few fundamental investigations are available in the literature about creep at early ages in traditional concretes, employing rheological test [7], ultrasonic test [8] or compressive test [15]. Further data and investigations have to be collected to compare the early-age creep behaviour of the printed material with that of traditional concrete [21]. In this context, we performed unconfined uniaxial compression tests to investigate the early-age creep response of a 3D printable mortar.

The aim of this paper is the identification of the critical aspects related to the early-age creep characterisation and modelling of printable mortars commonly used in 3DCP. Under a constant load, the earlyage mortar shows a total strain value which can be divided into two rates: an instantaneous and a delayed strain [15]. The former is mainly correlated to the elastic material response and to adopted printing process parameters (e.g., material pressure coming 
from the nozzle, printing speed, building rate), while the latter is mainly studied by surface thermodynamics theories that treat the cement paste as a two-phase material, i.e., characterized by micro-diffusion of both pore water and solid particles [22]. While pore water micro-diffusion is mainly correlated with settlement, consolidation and drying phase [15], solid particles micro-diffusion makes the solid phase more mobile, and therefore it is considered as the direct source of creep [22]. In fresh mortars the time range in which the delayed strain completely develops is drastically shorter than in hardened ones: further delayed deformations certainly continue to develop over time, but their magnitude is negligible for the phenomenon and the production process herein investigated. Accordingly, in the present paper authors suggest designating such delayed strain with the term "early-age creep".

To this scope, uniaxial compression tests are designed and carried out on cylindrical specimens of a cementitious printable mortar, in order to evaluate the material response under constant load at different resting times (i.e., 0, 15, 30 and $60 \mathrm{~min}$ ). Then, the experimental outcomes are used to calibrate a 1-D analytical model (i.e., the Burgers' model [23]), which considers the visco-elastic response exhibited by the printable mortar. In order to assess the robustness of the calibrated analytical model, further experimental tests are carried out on cylindrical specimens to simulate the step-by-step filament deposition process (i.e., increasing load). Total strain vs time curves were experimentally obtained by increasing the vertical load in three steps: in each step the load is maintained constant for a specific time interval and the mechanical response (including the creep effect) of the material under curing was assessed. Outcomes are then compared with analytical curves obtained by employing the calibrated Burgers' model. The analytical model can be a helpful tool to predict the actual vertical displacement of a $3 \mathrm{D}$ printed object during the automated stacking process, including also the cumulated non-instantaneous deformations; indeed, by assuming that each deposition step is characterised by an instantaneous constant load application (i.e., the weight of one filament) before the subsequent layers, the instantaneous and creep strain can be assessed. Therefore, the calibrated 1-D visco-elastic model is herein used to evaluate the vertical displacement of a 3D printed element, focusing on the influence of both the time gap between two subsequent layers and the 3D printable mortar stiffness.

\section{Materials and methods}

This section preliminary sets out a summary of the linear theory of viscoelasticity and the related existing analytical models. Subsequently, the authors describe the details of the printable mortar used in the experimental campaign as the reference mix in this research (already used in [24]). Finally in this section, authors introduce the iterative process designed to delineate governing testing parameters, such as the displacement rate on the first loading branch and the overall testing duration, in order to reduce the experimental effort satisfying both the instantaneous load application assumption and the full development of the early-age creep strain. Then, the experimental outcomes are used to calibrate the Burgers' analytical model, which considers both the elastic and viscous response exhibited by the printable mortar.

\subsection{Theoretical background}

The linear theory of viscoelasticity combines the different behaviours of a linear elastic and a viscous material within the hypothesis of homogeneous and isotropic continuum domain. The former can store elastic energy with no dissipation, while the latter (with reference to Newtonian viscous fluids) can dissipate energy without storing it [25]. The constitutive stress-strain relationship of a viscoelastic material is strongly dependent on the time variable, referred to the applied load or strain. In particular, the mechanical response over time is the result of both the loading history and the strain rate and it is characterised by the stress relaxation and creep effects. The overall mechanical response is modelled through simple spring-dashpot systems (for elastic and viscous behaviour, respectively), which are connected in series or parallel. The constitutive equation of the elastic component (i.e., the spring) is:

$\sigma=\varepsilon \cdot E$

where $\sigma[\mathrm{kPa}]$ is the stress, $\varepsilon[-]$ is the strain, and $E$ $[\mathrm{kPa}]$ is the Young's modulus. The viscous timedependent behaviour is modelled by the equation: 
$\sigma=\eta \cdot \frac{\mathrm{d} \varepsilon}{\mathrm{dt}}$

where $\eta[\mathrm{kPa} \cdot \mathrm{sec}]$ is the material viscosity, $d \varepsilon / d t$ $\left[\mathrm{sec}^{-1}\right]$ is the rate of change of strain, and $t[\mathrm{sec}]$ is the time of observation. Typically, more articulated analytical models can be constructed from Eqs. 1 and 2 to better reproduce the time-dependent response of viscoelastic materials. The Maxwell model is represented by an elastic spring and a viscous damper connected in series, as shown in Fig. 1a. Since both devices are subjected to the same stress, the model is also called the iso-stress model. By applying the derivative of total strain with respect to time and using Eqs. 1 and 2, the stress-strain relationship is governed by Eq. 3 (in which the subscript " $M$ " refers to the Maxwell device).

$\frac{\mathrm{d} \varepsilon}{\mathrm{dt}}=\frac{\sigma}{\eta_{M}}+\frac{1}{E_{M}} \cdot \frac{\mathrm{d} \sigma}{\mathrm{dt}}$

Such a model provides a stress behaviour that decays exponentially with the application of time permanent deformation (i.e., stress relaxation). The model does not accurately predict the creep effect under constant load $\sigma_{0}$ so the strain will increase linearly with time (with a slope of $\sigma_{0} / \eta_{M}$ ).

(a) Maxwell Model

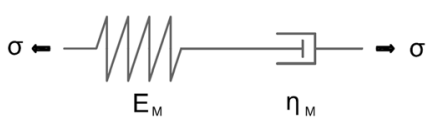

(b) Kelvin-Voigt Model

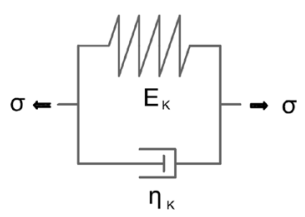

(c) Burgers Model

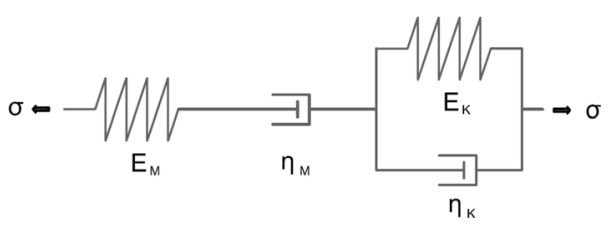

Fig. 1 Linear viscoelastic model: a Maxwell, b Kelvin-Voigt and c Burgers [26]
The Kelvin-Voigt model is represented by spring and damper arranged in parallel (see Fig. 1b); both devices are subjected to the same strain, so the total stress is governed by Eq. 2 (in which the subscript " $K$ " refers to the Kelvin-Voigt device).

$\sigma=E_{K} \cdot \varepsilon+\eta_{K} \cdot \frac{\mathrm{d} \varepsilon}{\mathrm{dt}}$

Through Eq. 4, it is possible to demonstrate that such a model is accurate to describe the creep effect: if a constant stress $\left(\right.$ i.e., $\left.\sigma(t)=\sigma_{0}\right)$ is applied the strain $\varepsilon$ will increase over time until a maximum value of $\sigma_{0} / E_{K}$, depending on the viscosity $\eta_{K}$. By contrast, the model has some limitations for stress-relaxation modelling: if a constant strain (i.e., $\varepsilon(t)=\varepsilon_{0}$ ) is applied, the model provides a constant value of the stress $\sigma=E_{K} \bullet \varepsilon_{0}$ without predicting its decrease.

A further elaboration of the spring-dashpot systems to describe viscoelastic materials is represented by the Burgers' model. It is composed of the Maxwell and Kelvin-Voigt models connected in series, as shown in Figs. 1c and 2. With the aim of getting the creep function, i.e., the analytical law of the time-dependent strain under constant applied load, the stress function has to be introduced through Eq. 5 .

$\sigma(t)=\sigma_{0} \cdot H\left(t-t_{0}\right)$

where $\sigma_{0}[\mathrm{kPa}]$ is the amplitude of the imposed stress at the time $t_{0}$ [sec] and $H(t)[-]$ is the unit step function. The creep function can be obtained by introducing the stress function $\sigma(t)$ (Eq. 5) in the Eqs. 3 and 4. Employing further analytical steps [26], it is possible to calculate the total strain $\varepsilon(t)$. As a result, the creep function of the Burgers' model can be obtained through the superposition principle, combining the response of Maxwell and Kelvin-Voigt model. The resulting analytical law for the strain variation over time is reported in Eq. 6.

$\varepsilon(t)=\frac{\sigma_{0}}{E_{M}}+\frac{\sigma_{0}}{E_{K}} \cdot\left(1-e^{-\frac{t}{t_{r}}}\right)+\frac{\sigma_{0}}{\eta_{M}} \cdot t$

where $t_{r}$ [sec] is the relaxation time (expressed as the ratio $\eta_{K} / E_{K}$ in the Kelvin-Voigt model). Equation 6 provides the total strain as the result of three components: the instantaneous strain, the delayed elastic strain and the irreversible creep strain (Fig. 2). In detail, the dampers with viscosity values of $\eta_{K}$ and $\eta_{M}$ allow modelling the so-called primary and secondary creep, respectively. The former tends to decrease over 


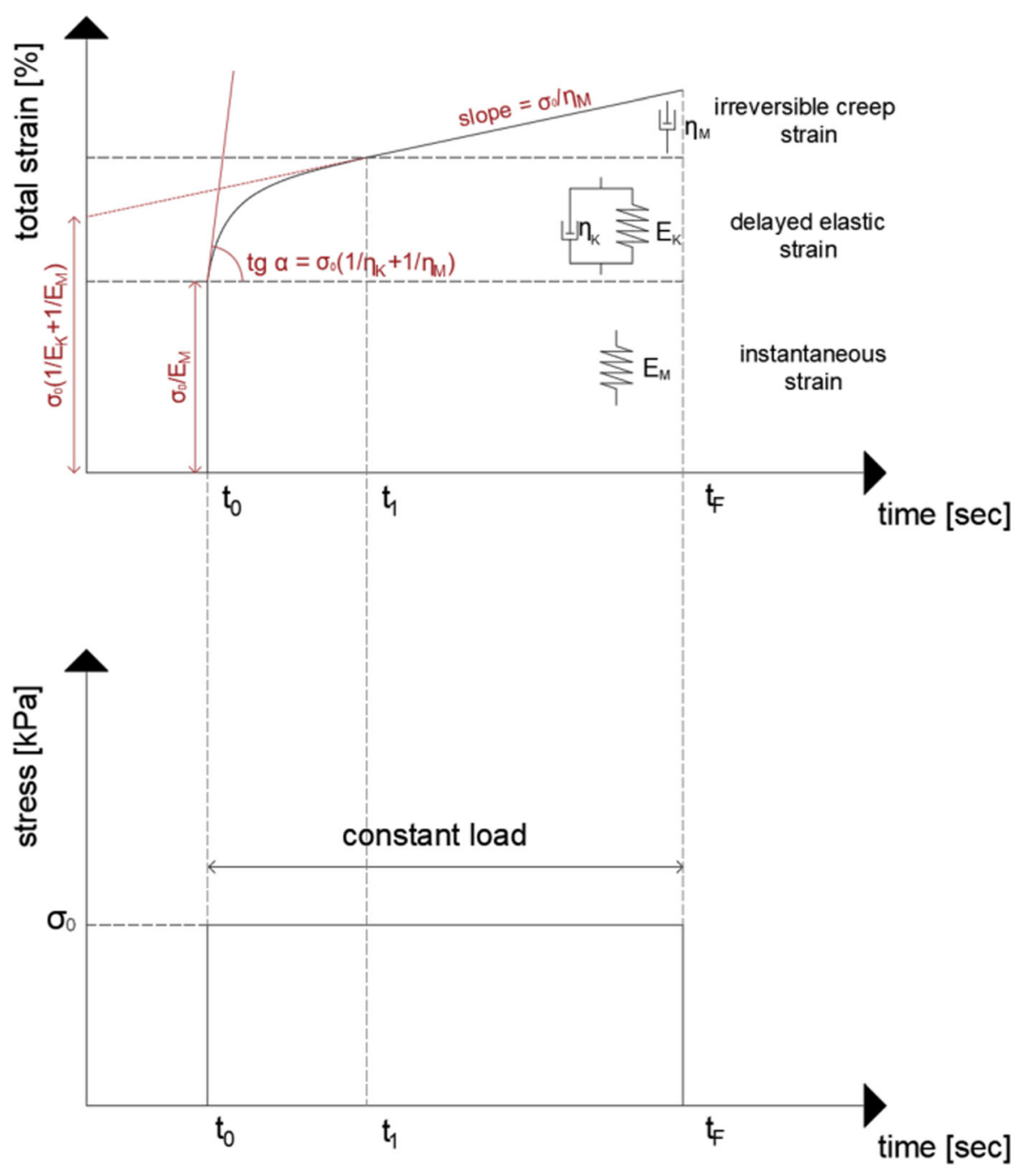

Fig. 2 Schematisation of the Burgers' model creep response

time, whereas the latter dominates the long-term deformation with the stationary creep rate $\sigma_{0} / \eta_{M}$. One of the main advantages of Burgers' viscoelastic model is the simple identification of four parameters from experimental time-dependent strain curves, as already done in literature for traditional Portland cement paste $[8,9]$. In this paper, such a model was chosen to describe the strain response recorded during experimental tests under a constant applied load. Since the issue to be faced is related to a monotone loading history of the $3 \mathrm{D}$ printing process, the unloading response is neglected. Specifically, the analytical model is herein used to predict the material response under the constant load related to a single-stacked layer. As a result, the total strain can be represented as the sum of two components: a time-independent strain, i.e., the instantaneous strain, and the time-dependent strain, i.e., delayed elastic strain and irreversible creep strain (as represented in Fig. 2).

\subsection{D printable mortar}

The experimental investigation on the viscoelastic behaviour was conducted on a $3 \mathrm{D}$ printable cementitious mortar utilised by the authors in [27] to fabricate steel-reinforced 3D printed beams. The same material was previously investigated by the authors through uniaxial compression tests in displacement control conditions: stress-strain relationships at different value of the resting time were obtained [24]. The 
mix was composed by sand (maximum particle size $4 \mathrm{~mm}$ ), $0.5 \%$ in weight of $18 \mathrm{~mm}$ polypropylene fibres (added to prevent plastic shrinkage-cracking during the early curing stage and after the deposition process), low water/cement ratio $(w / c=0.39)$ and a polycarboxylate superplasticizer $(\mathrm{SP}=0.10 \%$ in weight of cement) used to improve printability performances (i.e., pumpability and extrudability). Dry components were mixed with polypropylene fibres (140 rpm-30 s), subsequently the water was added mixing again the compound (140 rpm-60 s). Finally, the polycarboxylate superplasticizer was added gradually ( $285 \mathrm{rpm}-120 \mathrm{~s}$ ). The average cubic strength, $R_{\mathrm{cm}}$, of the mortar after 28 days of curing was determined over four specimens and was equal to 53.5 MPa with a standard deviation of 3.1 MPa [28]. The resulting material density was $2411 \mathrm{~kg} / \mathrm{m}^{3}$. Please refer to the mentioned papers for further details, also concerning the specimen preparation.

\subsection{Experimental method}

Early-age creep tests under uniaxial compression load were performed on cylindrical specimens, having the diameter, $d$, of $60 \mathrm{~mm}$ and the height, $h$, of $120 \mathrm{~mm}$ (Fig. 3a): the geometry was chosen to exclude size effects due to particle size distribution and to have $h /$ $d=2$, i.e., introducing diagonal shear failures. Specimens were tested by using an MTS electromechanical Universal Testing Machine, with $10 \mathrm{kN}$ capacity, at room temperature $T=22{ }^{\circ} \mathrm{C}$ and relative humidity $\mathrm{RH}=60 \%$. According to [24], such specimens were produced by means of a plastic 3D-printed openable mould (in order to provide good shape retention) and by using an internal nylon membrane (to effectively reduce the specimen-to-mould interface friction). After the casting procedure, the specimen was demoulded and the membrane removed; in this way, the curing took place in the same drying condition for both the specimen and the 3D printed object.

Figure $3 \mathrm{~b}$ illustrates the loading history which was composed of two parts: an increasing linear branch up to the target load, $\mathrm{F}_{0}$ (i.e., $8 \mathrm{~N}$, the weight of the specimen), performed in the displacement-control condition in the range of time $0-t_{0}$; and a horizontalconstant over time-branch, performed in the loadcontrol condition in the range of time $t_{0}$ and $t_{F}$. The displacement vs time testing record was converted in a total strain vs time curve. In detail, the total strain $\varepsilon_{t o t}^{E x p}$ was obtained by dividing the displacement of the loading head by the specimen height; whereas the target stress $\sigma_{0}$ was obtained by dividing the recorded target force $F_{0}$ by the updated cross-sectional area, assessed according to the equation provided in Section 8 of ASTM D2166/D2166M [29].

To avoid non-controlled interferences with time dependent material behaviour, the overall testing procedure was iteratively calibrated in order to satisfy two constraints:

(a) The Displacement Rate (DR) in the increasing linear branch up to the target load, $F_{0}$ must be high enough to consider the load instantaneously applied (the relaxation time $t_{r}$ must be greater than the time $t_{0}$, so that viscous strains can be considered negligible in the time range 0 $\left.t_{0}\right)$

(b) The whole test duration $\left(T_{\text {tot }}=t_{0}+t_{F}\right)$ must permit the full development of the early-age creep strain.

For the sake of clarity, the logic scheme of the iterative process is reported in Fig. 4.

As schematically reported in Fig. 4, (i) three specimens were prepared according to [24] and (ii) tested with an initial value of DR and $T_{\text {tot }}$, selecting a specific resting time among $t^{*}=0,15,30$ and $60 \mathrm{~min}$. Subsequently, (iii) once the average total strain vs time curve was experimentally obtained, (iv) the Burgers' model parameters were assessed exploiting the following equations:

$\varepsilon\left(t_{0}\right)=\frac{\sigma_{0}}{E_{M}}$

$\lim _{t \rightarrow \infty} \dot{\varepsilon}(t)=\frac{\sigma_{0}}{\eta_{M}}$

$\varepsilon\left(t_{1}\right)-\varepsilon\left(t_{0}\right)=\sigma_{0} / E_{K}$ where $\frac{\mathrm{d} \varepsilon}{\mathrm{dt}}$ $=$ constant for $t \geq t_{1}$

$\operatorname{avg}\left(\frac{\varepsilon_{\text {tot }}^{\operatorname{Exp}}(t)-\varepsilon_{\text {tot }}^{\text {BURGERS }}\left(t, t_{r}\right)}{\varepsilon_{\text {tot }}^{\operatorname{Exp}}(t)}\right)<0.01 \%$

Equation 7 allows assessing the stiffness value of the Maxwell spring, with the simplified initial assumption (to be checked with subsequent iterations) that at time $t_{0}$ only an elastic instantaneous strain 
(a)
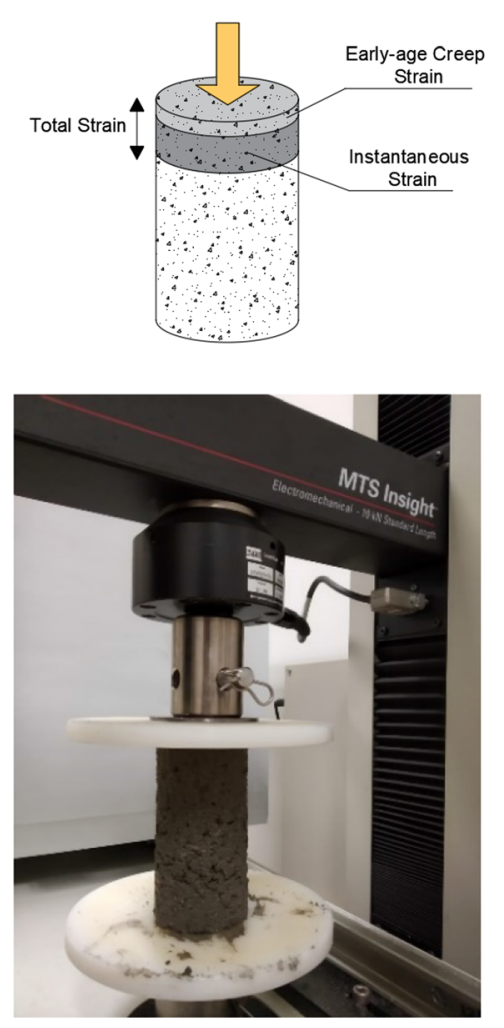

Fig. 3 a Sketch and tested specimen and $\mathbf{b}$ loading history

arises, i.e., the viscous contribution is neglected. Equation 8 requires that, after $t_{0}$, the slope of the experimental curve is equal to the $\sigma_{0} / \eta_{M}$ ratio for long-lasting observation times: such a condition allows the determination of the viscosity value of the Maxwell damper $\eta_{M}$ in Fig. 1a. Equation 9 allows evaluating the stiffness of the Kelvin-Voigt spring $\mathrm{E}_{\mathrm{K}}$ in Fig. 1b, which defines the maximum value of the early-age creep strain. Equation 10 is used for the calibration purpose, which allows finding the relaxation time value $t_{r}$ (expressed as the ratio $\eta_{K} / E_{K}$ in the Kelvin-Voigt model) that minimises the relative error between experimental and analytical curve data; in detail, the matching was done in the range $t_{0}-t_{F}$ : the goal-seeking tool was used to find the $t_{r}$ value, which substituted in Eq. 6 provides an average scatter of $0.01 \%$ (i.e., $\varepsilon_{\text {tot }}^{B U R G E R S}-\varepsilon_{\text {tot }}^{E x p}$ ). Once obtained the Burgers' parameter values, it is possible to verify that (v) the value of the time $t_{0}$ is negligible if compared with the relaxation time of the material (i.e., condition (b)

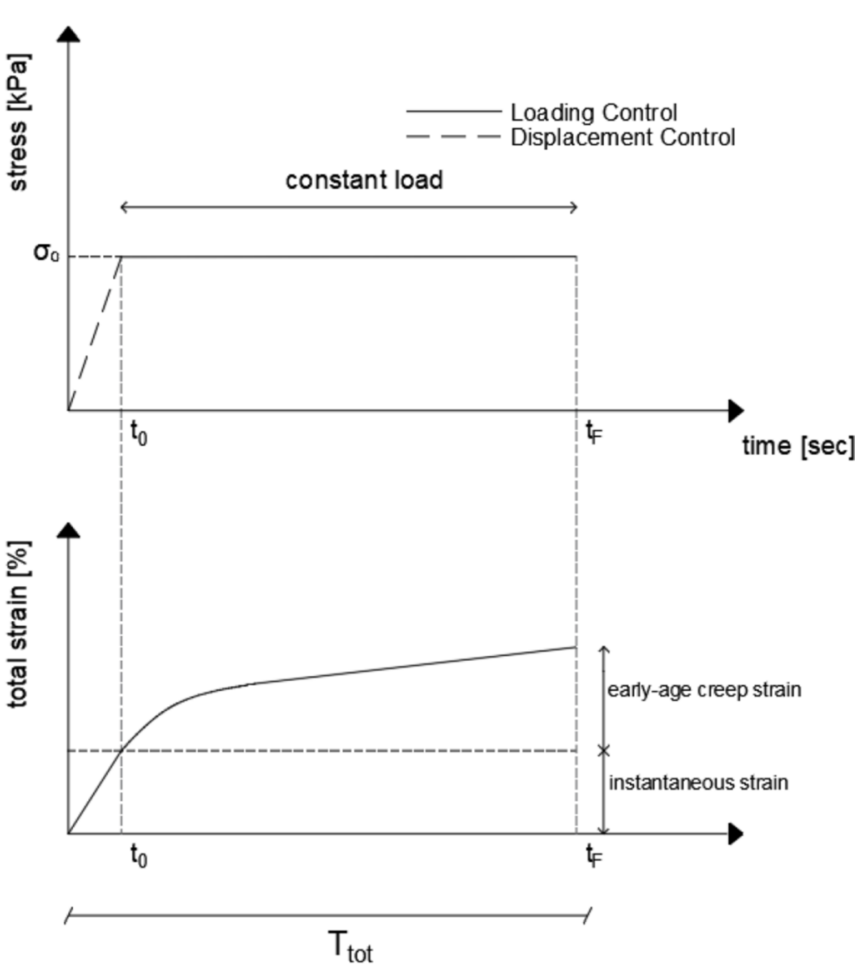

(a) above) and that (vi) the strain is fully developed (i.e., condition (b) above).

Table 1 contains a summary of the experimental tests carried out. Each iteration was executed for different values of the mortar ages (i.e., 0, 15, 30 and $60 \mathrm{~min}$ ) in order to obtain the evolution over time of the resulting viscoelastic parameters that, in turn, could be useful for modelling the evolution of the printing process. Indeed, by determining the Burgers' model parameters for different resting times, the total strain accumulated over time by each printed layer due to the building up process can be accurately quantified. Each specimen set is identified by the acronym "DRxx-Tyy-tzz", where: "DRxx" represents the displacement rate in $\mathrm{mm} / \mathrm{min}$, "Tyy" indicates the test duration in seconds, and "tzz" is the resting time in minutes. 


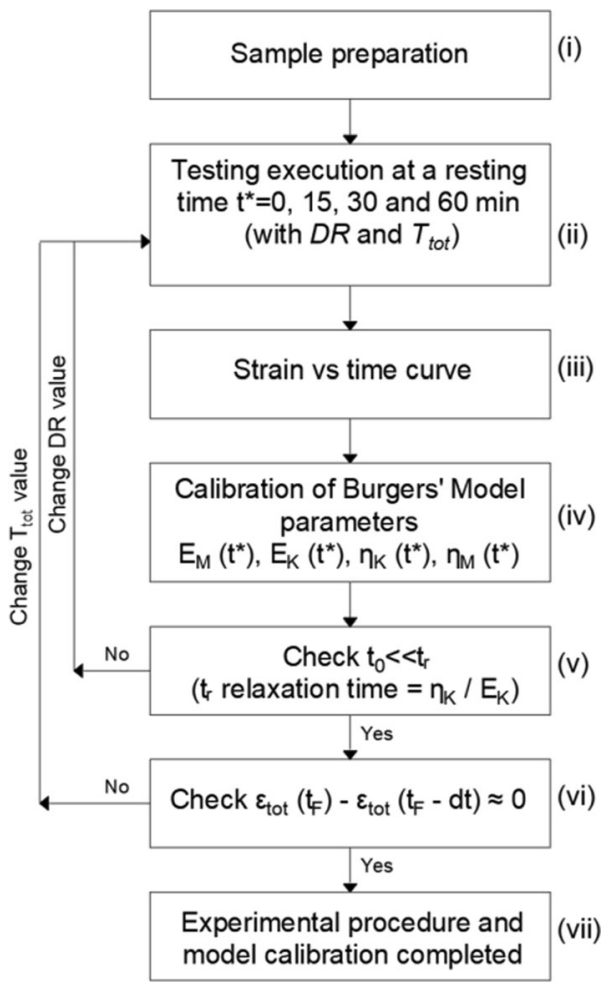

Fig. 4 Logic scheme of the iterative process for experimental procedure and Burgers' model calibration

\section{Experimental results and Burgers' model calibration}

By iterating the process showed in Fig. 4, it was possible to calibrate the analytical Burgers' model for each value of the resting time (i.e., $0,15,30,60 \mathrm{~min}$ ), characterising the evolution over time of the viscoelastic material behaviour. The iterative process allowed obtaining the testing parameter values suitable to investigate the early-age creep for the tested fresh mortar. Starting from $\mathrm{DR}=3 \mathrm{~mm} / \mathrm{min}$ and $T_{\text {tot }}=300 \mathrm{~s}$, the iteration process converged to the calibrated values of $\mathrm{DR}=30 \mathrm{~mm} / \mathrm{min}$ and
$T_{\text {tot }}=500 \mathrm{~s}$. In detail, the optimal and calibrated displacement rate adopted in this study is congruent with other works available in literature focused on cement-based printable mortar [30-32]. In the following, the experimental results and the calibrated parameters of the Burgers' model are reported and discussed. The average force vs time diagram representative of the different specimens is reported in Fig. 5, in which it is possible to observe that a stiffer material (e.g., after $60 \mathrm{~min}$ of resting time) is able to reach the target load $(8 \mathrm{~N})$ in a shorter time, once the DR is defined.

In Fig. 6, the total strains $\varepsilon_{t o t}$ are plotted as a function of the testing time, from 0 to $500 \mathrm{~s}$, for each value of mortar ages $(0,15,30$ and $60 \mathrm{~min})$ investigated and for each tested specimen (dotted curves represent the different samples whereas continuous ones the average curves). It is worth noting that the total strain $\varepsilon_{t o t}$ decreases from $2.2 \%$ (at $0 \mathrm{~min}$ ) to $0.6 \%$ (at $60 \mathrm{~min}$ ) and its increment asymptotically tends to zero after about $300 \mathrm{~s}$ of testing time. As aforementioned, under a constant load, fresh mortars exhibited both an instantaneous and early-age creep strain. It is possible to notice that early-age creep deformation in time, recorded for the investigated 3D printable mortar, reflect typical creep deformation trend of hardened traditional concrete [33, 34]; however, conversely to the creep effect in traditional concrete, early-age creep strains develop in terms of seconds. Finally, fresh mortar experimental curves depicted in Fig. 6 show an increasing slope of the first branch (i.e., representing the loading phase), passing from 0 to $60 \mathrm{~min}$.

Figure 7 illustrates the experimental average total strain vs time curve, whereas Fig. $7 \mathrm{~b}$ summarizes the amount of the elastic and early-age creep strain obtained from experiments. Both curves decrease in time due to the material hardening evolution: the early-age creep strain represents a pronounced amount of the total vertical deformation, with a value of $1.1 \%$

Table 1 Test matrix

\begin{tabular}{llll}
\hline \multicolumn{2}{l}{ Early-age creep test (calibration procedure) } & \\
\hline Variables & Acronym & & Target load \\
\hline Displacement rate [mm/min] & DR $x x$ & DR $x x$-Tyy-tzz & $8 \mathrm{~N}$ \\
Test duration [sec] & Tyy & \\
Resting time [min] & $\mathrm{t} z z$ & \\
Total specimens for iteration & 3 & \\
\hline
\end{tabular}



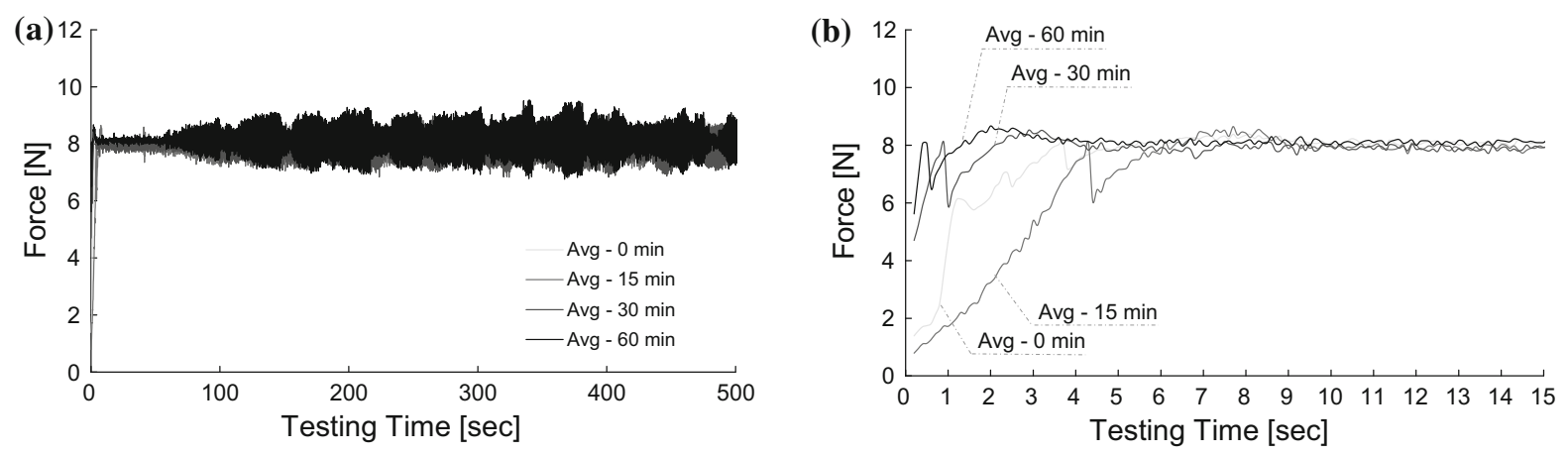

Fig. 5 Recorded testing head force for a Displacement Rate (DR) equal to $30 \mathrm{~mm} / \mathrm{min}$ : a total testing time, b zoom detail
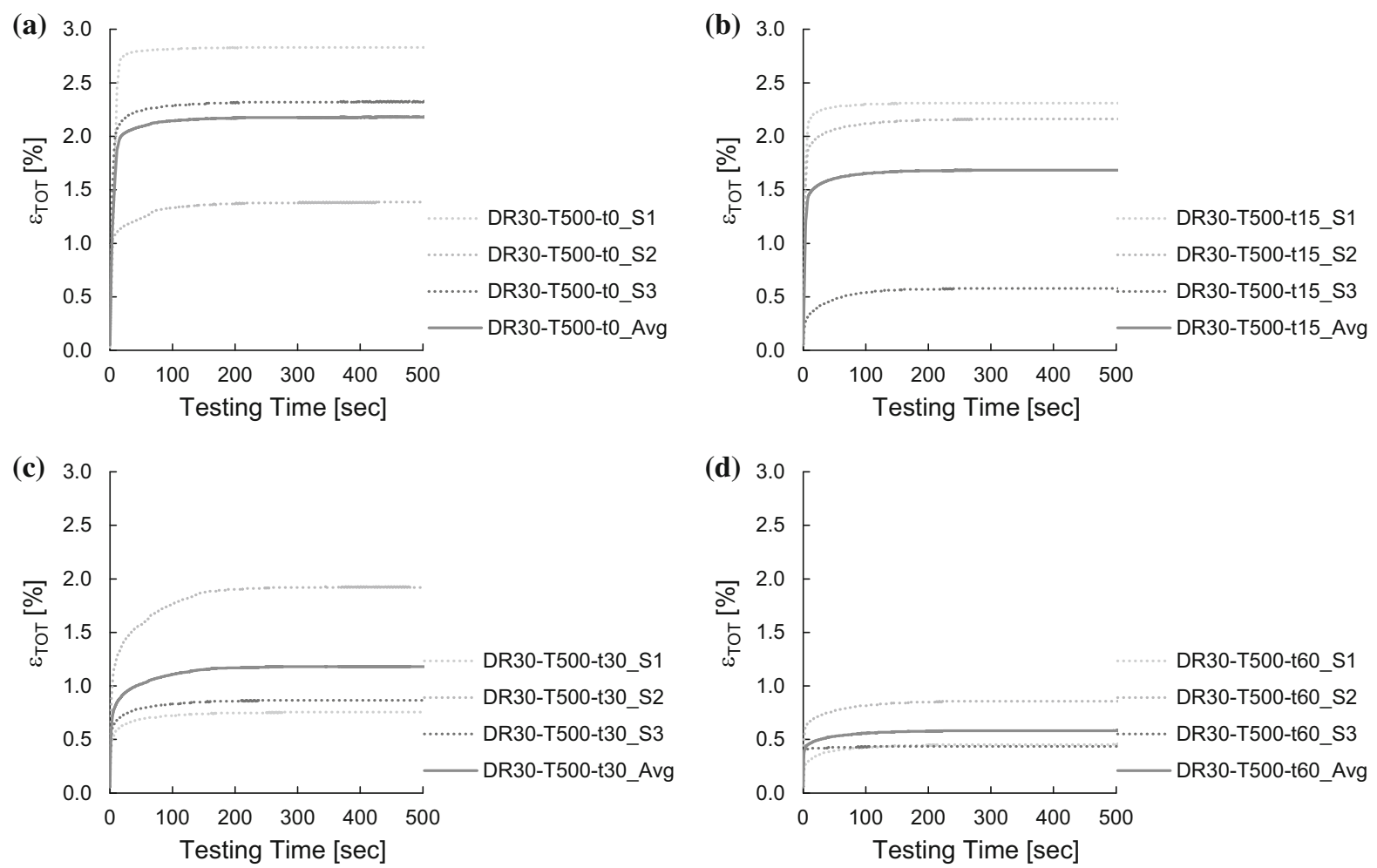

Fig. 6 Individual and average experimental total strain VS testing time for each considered resting time: a 0, b 15 , c 30 and d 60 min

and $0.2 \%$ at 0 and $60 \mathrm{~min}$, respectively. It is worth noting that, up to $30 \mathrm{~min}$ (i.e., where it is possible to find the transition from viscous to brittle shear failure behaviour) the absolute value of early-age creep is large. The calibration of the Burgers' model parameters as a function of the resting time of the printable mortar was achieved by employing the experimental average total strain vs time curves plotted in Fig. 7a, and using Eqs. 6, 7, 8 and 9. Primarily, the time $t_{0}$ was found as the testing time at which the strain rate was less than $0.1 \%$ compressive strain/sec. At time $t_{0}$, the corresponding strain value $\varepsilon\left(t_{0}\right)$ was assumed as the purely instantaneous elastic component. The time $\mathrm{t}_{0}$, as expected, strongly depends on the resting time (see also Fig. 5): at earlier ages, the material is less stiff, so the time $t_{0}$ is higher; while for larger resting times, the function of time $t_{0}$ reduces up to zero as asymptotic limit (Fig. 8).

Similarly, the values of the spring stiffness, $E_{M}$ and $E_{K}$, and the value of the damper viscosity, $\eta_{K}$, were 

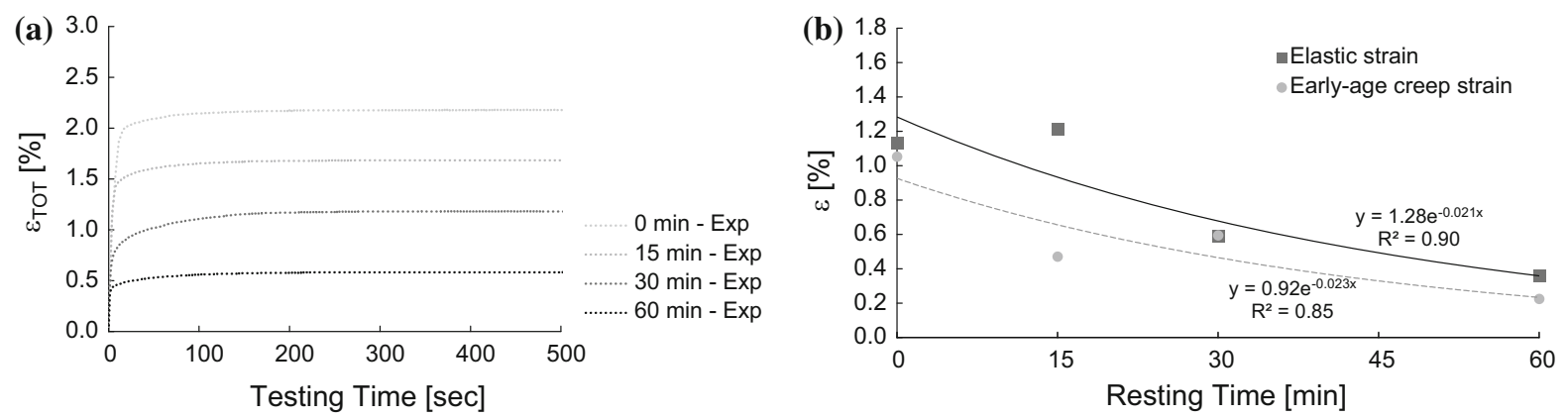

Fig. 7 Experimental strain: a average total strain vs time curves, b elastic vs average early-age creep strain for each considered resting time: a 0 , b 15 , c 30 and d $60 \mathrm{~min}$

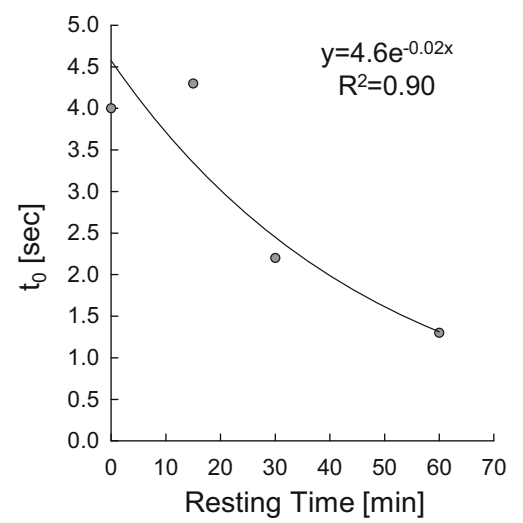

Fig. 8 Time t0 as a function of the material hardening

determined from the average curves of Fig. 7. As a result, a time-dependent law was found for each one of the Burgers' model parameter by the linear fitting of experimental data, as shown in Fig. 9. From the analysis of the values obtained, both stiffness values (i.e., Maxwell and Voigt stiffnesses, $E_{M}$ and $E_{K}$ ) increase with the resting time (Figs. 5a, b); this means that both instantaneous and delayed elastic strain (Fig. 2) tend to decrease with the hydration process. Furthermore, the relaxation time increases over time (Fig. 9c): indeed, the evolution of the cement hydration leads to the transition from a viscous-fluid to an elastic-plastic solid behaviour, to the extent that the time required to reach the equilibrium state after a stress/strain perturbation tends to grow. Moreover, from Fig. 7a it is clear that the last part of all timestrain experimental curves has a flat slope.

As a result, Eq. 8 leads to a very high value of the Maxwell viscosity $\eta_{M}$, which means that the Maxwell damper can be neglected in this study and for the observation time selected.

$$
E_{M}(t)=9.6 \cdot t+178.8[\mathrm{kPa}]
$$

(a)

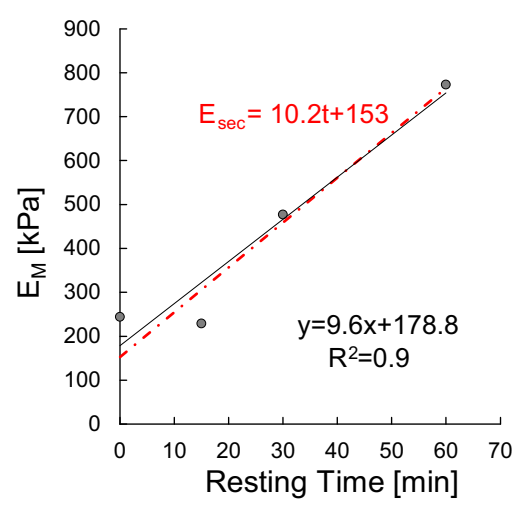

(b)

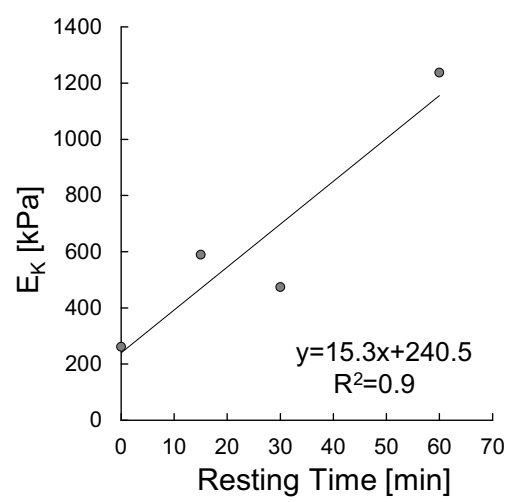

(c)

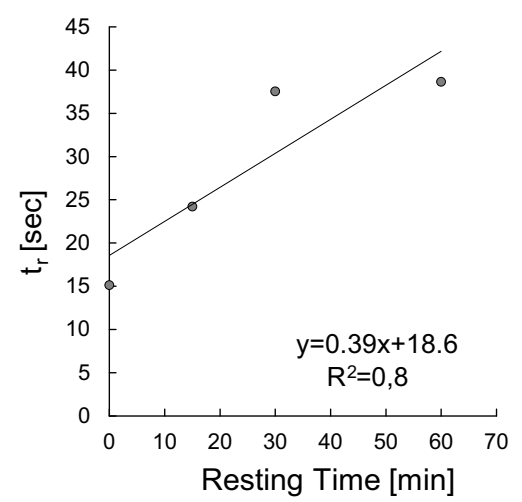

Fig. 9 Time-dependent laws of Burgers' parameters: a Maxwell and b Voigt stiffness, and c relaxation time 
$E_{K}(t)=15.3 \cdot t+240.5[\mathrm{kPa}]$

$t_{r}(t)=0.39 \cdot t+18.6[\mathrm{sec}]$

The resulting analytical total strain vs time curves obtained through the Burgers' parameters are reported in Fig. 10, along with the corresponding experimental curve.

By comparing the time-dependent law of the Maxwell stiffness $E_{M}(t)$ (Eq. 11) with the timedependant elastic secant modulus obtained from the experimental campaign carried out on the same 3D printable mortar in [24] (Eq. 14), it was possible to observe a good matching between the corresponding total strain vs time evolution curves (see red dashed line in Fig. 9a).

$E_{\mathrm{sec}}(t)=10.2 \cdot t+153[\mathrm{kPa}]$ from $[24]$

It is worth noting that the former was obtained through an analytical calibration based on experimental early-age creep strain curves obtained for different resting times, whereas the latter was determined as the secant modulus achieved by uniaxial compression tests in displacement control (i.e., from stress and strain measurements). Consequently, it appears that the time evolution of the measured Young's modulus in compression of the printable mortar roughly correspond to the elastic component of a viscoelastic system represented by the Burgers' model and, specifically, by the Maxwell spring.

\section{Analytical model validation}

After a proper setting-up of the early-age creep testing procedure, the Burgers' model parameters were

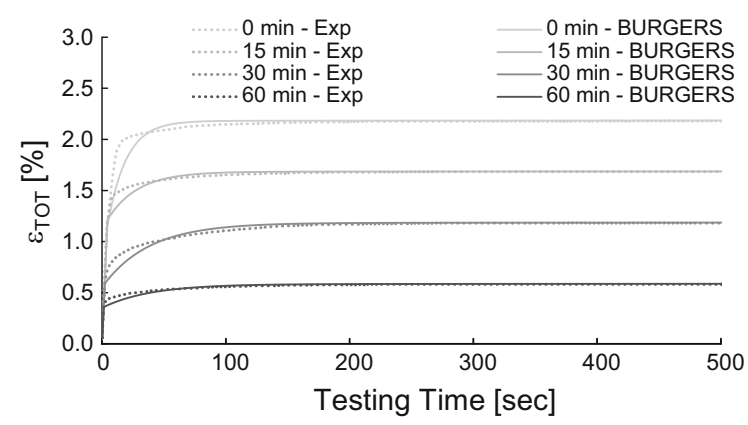

Fig. 10 Average experimental VS analytical temporal evolution of total strain curves calibrated, resulting in a good agreement between experimental average curves and the analytical strain prediction over time (Fig. 10). The viscoelastic Burgers' model and corresponding analytical total strain vs time curves can be used to predict the instantaneous and the viscous compressive strain of each stacked cementitious mortar layer, created during the step-bystep layered extrusion process. However, the printing parameter variability (i.e., layer height, building rate, time gap) can be significantly dependent on the geometry of the printed object as well as by the printing equipment adopted. In order to assess the robustness of the calibrated model in terms of compression strain/displacement prediction during printing, further tests were designed and carried out experimentally to simulate the step-by-step deposition process. In particular, cylindrical specimens of dimensions $60-120 \mathrm{~mm}$ (diameter and height, respectively) were increasingly loaded in three different phases to simulate three stacking steps: for each increment the weight of the subsequent stacked layer acting on the first reference layer is applied and then maintained constant for $300 \mathrm{~s}$ in order to record creep at such stacking step, as shown in Fig. 11.

The time between each loading step was fixed equal to $300 \mathrm{~s}$. The $3 \mathrm{D}$ printable mortar was loaded up to the target value (i.e., $8 \mathrm{~N}$ ) with a strain-rate value of $0.025 \mathrm{~min}^{-1}$. Even in these experiments, different resting time values of $0,15,30$ and $60 \mathrm{~min}$ were investigated, and three specimens were tested for each specimen set. The corresponding analytical total strain vs time curves were generated from Burgers' parameters previously calibrated (see Eqs. 11, 12 and 13 and Fig. 9), as explained in Sect. 3. From those values, the analytical curve was constructed with the simple assumption that the parameters remain constant during the single-step depending only on the resting time $t$ at which the load is applied; in that way, the total vertical strain was assessed applying the Eq. 6:

$\varepsilon(t)=\frac{\sigma_{0}}{E_{M}(t)}+\frac{\sigma_{0}}{E_{K}(t)} \cdot\left(1-e^{-\frac{t}{t_{r}(t)}}\right)$

where $\sigma_{0}$ is the increment of the vertical stress at each step and $t$ is the testing time, which correspond to the resting time. The comparison between experimental average total strain vs time curves (dashed lines in Fig. 12 including the experimental scatter as a coloured region around the average curves) and the analytical prediction according to the viscoelastic 

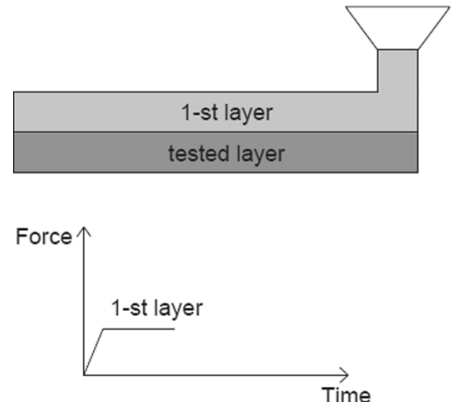
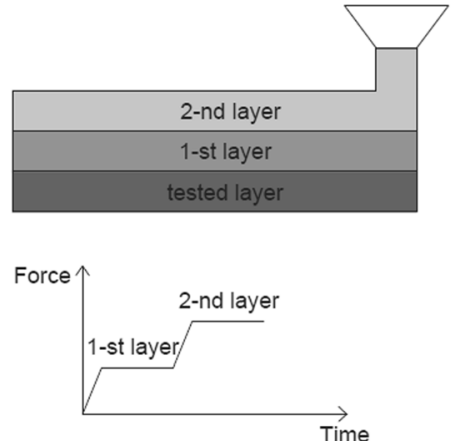
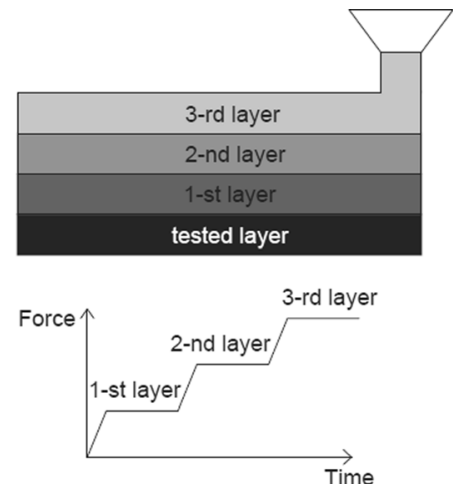

Fig. 11 Simulation of one-, two- and three-layer stacking sequence which was repeated for different resting times

Burger's model (continuous lines) is reported in Fig. 12. The material loaded at 0 min (i.e., green curves) displays a total vertical strain at $900 \mathrm{~s}$ of 6.8 and $6.3 \%$ (i.e., experimental and simulated values, respectively). Such values decrease with curing time, reaching 1.8 and $1.6 \%$ at $60 \mathrm{~min}$ (i.e., red curves).

The maximum relative percentage error (i.e., $\left.\frac{\varepsilon_{\text {tot }}^{\text {BURGERS }}-\varepsilon_{\text {tot }}^{E x p}}{\varepsilon_{\text {tot }}^{E x p}} \cdot 100[\%]\right)$ between the analytical prediction and the experimental outcomes is higher at earlier ages in correspondence with the first loading step (i.e., at $0 \mathrm{~min}$ is equal to $-25 \%$ ); respectively, the experimental scatter assumes the highest value that is approximately $1.47 \%$ (see the filled area around the experimental curves in Fig. 12). The analytical model provides a good prediction on the long-term strain: the maximum relative percentage error computed at $900 \mathrm{~s}$ (i.e., after the three loading steps) is equal to approximately $+8,-11,+4$ and $+8 \%$ (within the experimental scatter) at $0,15,30$ and $60 \mathrm{~min}$, respectively.
Experimental scatter and the relative percentage error could be reduced by adopting a more accurate system measurement of the vertical deformation, as already done in other experimental investigations [30, 32, 35].

Based on the satisfactory agreement obtained in Fig. 12, the Burgers' model was further exploited to predict the total vertical displacement of printed filaments during a simulated case study based on the layered extrusion process. In detail, the total vertical displacement was additively computed from the analytical assessment of the vertical strain under the self-weight of the progressively deposited concrete layers. In the simulated case study, the layer height, $h_{\text {layer }}$, was fixed equal to $10 \mathrm{~mm}$, therefore the increment of vertical compressive stress $\Delta \sigma$ at each deposition step acting on the first layer was:

$\Delta \sigma=\rho \cdot g \cdot h_{\text {layer }}$

where $\rho$ is the material density $\left(2411 \mathrm{~kg} / \mathrm{m}^{3}\right)$, and $g$ is the acceleration of gravity. Said "- $\mathrm{i}$ " the generic
Fig. 12 Simulation of the stacking process: Experimental VS Analytical total strain vs time curves

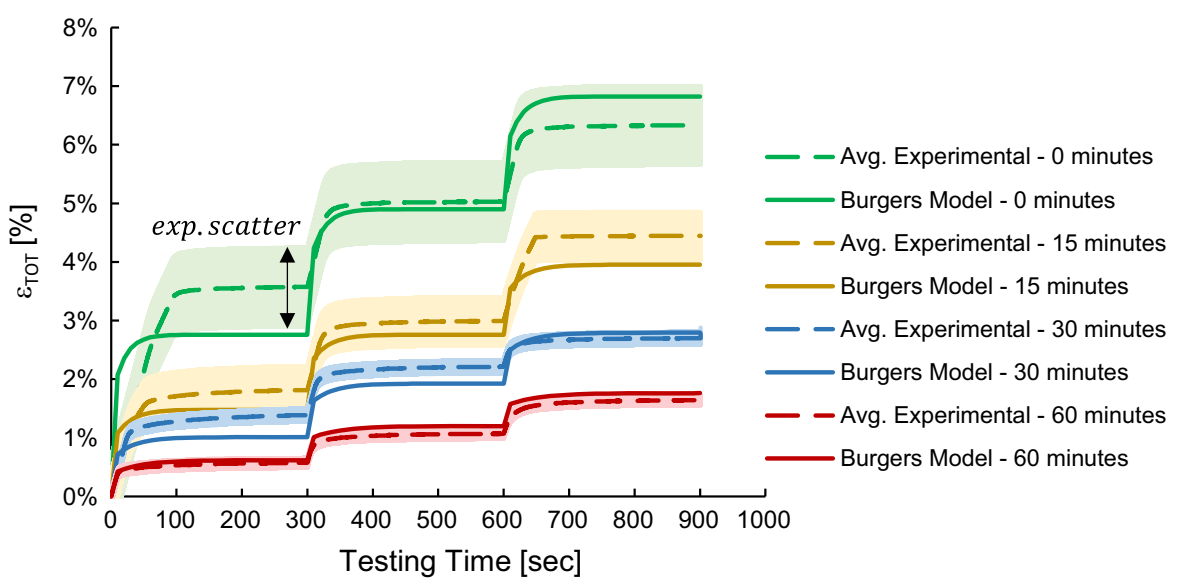


deposition step, the resting time of the $\mathrm{j}$-th stacked layer (i.e., $t_{j}^{i}$ ) is:

$t_{j}^{i}=(i-j) \cdot t_{\text {time gap }}$ with $i \geq j$

where $t_{\text {time gap }}$ is the time gap between two consecutive layers. The total strain of the $j$-th stacked layer due to the self-weight of the $i$-th deposited filament, i.e., $\varepsilon_{j}^{i}$, was calculated through Eq. 6. The only contribution of Maxwell and Kelvin-Voigt springs was considered in order to assess the final value of the total strain (rather its evolution), i.e., the summation of the instantaneous and creep strain:

$\varepsilon_{j}^{i}\left(t_{j}^{i}\right)=\frac{\sigma_{0}}{E_{M}\left(t_{j}^{i}\right)}+\frac{\sigma_{0}}{E_{K}\left(t_{j}^{i}\right)}$

in which $\sigma_{0}=\Delta \sigma=0.219 \mathrm{kPa}$ is a constant value and the Burgers' parameters were assessed according to Eqs. 11 and 12 at $t=t_{j}^{i}$.

Once the vertical strain of each $j$-th filament for each deposition step is assessed, employing the superposition principle, it is possible to calculate the accumulated vertical displacement of a printed element with the following double summation (Eq. 15).

$d=\sum_{i=1}^{n} \sum_{j=1}^{n} \varepsilon_{j}^{i}\left(t_{j}^{i}\right) \cdot h_{j}^{i}$

where $\varepsilon_{j}^{i}\left(t_{j}^{i}\right)$ is the total vertical strain of $j$-th filament at $i$-th deposition step, and $h_{j}^{i}$ is the updated value of the layer height taking into account the increasing value of the deformation.

The vertical displacement depends on several technological parameters as well as the material properties, the stress imposed by a single stacked filament and the total height of the object. Assuming a straight 3D printed wall composed of 50 layers, $500 \mathrm{~mm}$ high, the total vertical displacement computed on the whole element was assessed by varying the time gap between subsequent layers and the material stiffnesses, trying to take onto account different practical scenarios. In detail, according to current researches [4, 36-38], time gap values of 30, 120,300 and $900 \mathrm{~s}$ and an increment of material axial stiffness of 25,50 and $100 \%$ (i.e., $E_{M}$ and $E_{K}$ ) at time 0 min were considered.

Figure 13 summarises the analytical results of the vertical displacement prediction employing the

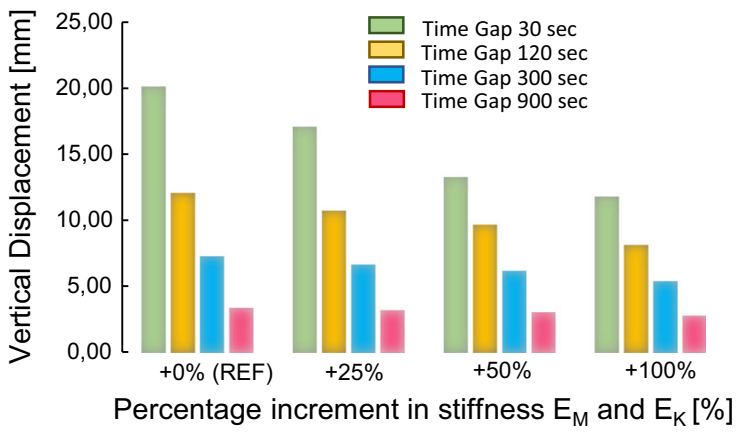

Fig. 13 Vertical displacement prediction-Burgers' Model

Burgers' model. It is worth highlighting that for the material under investigation, the main parameter that influences the vertical displacement is the time gap; indeed, the displacement of the reference mix (i.e., $+0 \%$ (REF) in Fig. 13) is equal to $20.1 \mathrm{~mm}$ for the lowest value of time gap (30 s) and drops to $3.4 \mathrm{~mm}$ when the time gap is $900 \mathrm{~s}$ (i.e., with a reduction of about $83 \%$ ). Moreover, in the case of stiffer mortars, e.g. the stiffness increment is $100 \%$, the vertical displacement computed with a time gap of $30 \mathrm{~s}$ is $11.8 \mathrm{~mm}$, i.e., reduction of about $41 \%$.

\section{Conclusion}

Printable cementitious mortars typically used in the layered extrusion process behave as viscoelastic material: generally, this means that the mechanical response to an external stimulus (i.e., imposed strain or load) can not be completely instantaneous, but part of it can be delayed. At each step of the deposition process, the single layer is subjected to a constant increment of vertical stress. Hence, according to the theoretical creep response, in its early age (which approximately corresponds to the printing time) the material exhibits both an instantaneous and a viscous vertical strain. In detail, the viscous strain component at early age develops quickly, and it could be a significant percentage of the overall vertical strain as it accumulates over time. As a consequence, for tall structures, a proper investigation of such phenomenon becomes more important, especially during the printing process. Therefore, an accurate prediction of the vertical strain is useful to calibrate the robotics motion and the dimensional/quality control. Moreover, another critical experimental challenge is the 
hardening of the cement paste: it is necessary to develop a reliable viscoelastic constitutive model accounting for this phenomenon. In this context, the Burgers' model could represent a satisfactory way to model the creep strain response over time of the earlyage material, taking into account both mechanical and viscous material properties. The Burgers' model is defined if the four corresponding parameters are known: since the material hardens at a fresh state, it is necessary to define the Burgers' parameters as a function of the resting time. A good prediction of the total strain depends on the reliability of the material mechanical model obtained by experimental data fitting over time.

Since the temporal evolution of mechanical properties is a new focus in 3DCP, in this work the earlyage creep effect was studied through an experimental campaign carried out on cementitious printable mortar in the fresh state and at different resting times $(0,15$, 30 and $60 \mathrm{~min}$ ). In detail, the loading history was composed of a loading ramp up to a target force value, which was maintained constant in order to record the vertical displacement due to the early-age creep effect. The testing parameters, i.e., the displacement rate of the first loading branch and the testing duration, were chosen through an iterative process in order to satisfy: the instantaneous load application assumption and the full development of the early-age creep strain. Experimental outcomes, i.e., total strain vs time curves at 0 , 15,30 and $60 \mathrm{~min}$, were used to calibrate the Burgers' analytical model: for each parameter, a time-dependent law was achieved. The time-dependent law of the Maxwell stiffness has a good match with the elastic secant modulus obtained in [24]. Validation of the calibrated model was achieved through further experimental tests that simulate the step-by-step deposition process: the analytical model provides a good prediction on the long-term strain, where the differences fall within the experimental scatter. Based on the calculations, the Burger's model tends to be a useful tool to evaluate the overall vertical displacement of a $3 \mathrm{D}$ printed element: a sensitivity analysis was performed to assess the influence of the time gap and the material stiffness. Further developments should be made to consider the variability in mix composition and correlated material curing properties. The diversification in $3 \mathrm{D}$ concrete printing technologies (e.g., building rates, time gap, pressure during extrusion, nozzle characteristics) has to be considered in future research: the fabrication of full-scale 3D concrete printed objects, equipping setup with optic measurement tools to improve the accuracy of the results, should provide data useful to validate the employment of the Burgers' model and the early-age creep quantification.

Authors' contributions LE: Conceptualization, Methodology, Writing. LC: Methodology, Experimental investigation, Writing. CM: Conceptualization, Methodology, Supervision. DA: Supervision. FA: Supervision, Writing.

Funding Open access funding provided by Università degli Studi di Napoli Federico II within the CRUI-CARE Agreement. The experimental investigation was supported by Regione Lombardia through the "TPro.SL-Tech Profiles for Smart Living” project, ID: 379384.

\section{Declarations}

Conflicts of interest Authors declare that they have no conflict of interest.

Open Access This article is licensed under a Creative Commons Attribution 4.0 International License, which permits use, sharing, adaptation, distribution and reproduction in any medium or format, as long as you give appropriate credit to the original author(s) and the source, provide a link to the Creative Commons licence, and indicate if changes were made. The images or other third party material in this article are included in the article's Creative Commons licence, unless indicated otherwise in a credit line to the material. If material is not included in the article's Creative Commons licence and your intended use is not permitted by statutory regulation or exceeds the permitted use, you will need to obtain permission directly from the copyright holder. To view a copy of this licence, visit http://creativecommons.org/licenses/by/4.0/.

\section{References}

1. Buswell R, de Silva WRL, Jones SZ, Dirrenberger J (2018) 3D printing using concrete extrusion: a roadmap for research. Cem Concr Res. https://doi.org/10.1016/j. cemconres.2018.05.006']

2. Khan MS, Sanchez F, Zhou H (2020) 3-D printing of concrete: beyond horizons. Cem Concr Res 133:106070. https://doi.org/10.1016/j.cemconres.2020.106070

3. Kruger J, Cho S, Zeranka S et al (2020) 3D concrete printer parameter optimisation for high rate digital construction avoiding plastic collapse. Compos B Eng 183:107660. https://doi.org/10.1016/j.compositesb.2019.107660

4. Roussel N (2018) Rheological requirements for printable concretes. Cem Concr Res. https://doi.org/10.1016/j. cemconres.2018.04.005 
5. Perrot A, Rangeard D, Pierre A (2015) Structural built-up of cement-based materials used for 3D-printing extrusion techniques. Mater Struct 49:1-8. https://doi.org/10.1617/ s11527-015-0571-0

6. Comminal R, Silva W, Andersen T, et al (2020) Influence of processing parameters on the layer geometry in 3D concrete printing: experiments and modelling. In: RILEM International Conference on Concrete and Digital Fabrication. Springer, Cham, pp. 852-862. https://doi.org/10.1007/9783-030-49916-7_83

7. Qian Y, Kawashima S (2016) Use of creep recovery protocol to measure static yield stress and structural rebuilding of fresh cement pastes. Cem Concr Res 90:73-79. https:// doi.org/10.1016/j.cemconres.2016.09.005

8. Sun Z, Voigt T, Shah SP (2006) Rheometric and ultrasonic investigations of viscoelastic properties of fresh Portland cement pastes. Cem Concr Res 36:278-287. https://doi.org/ 10.1016/j.cemconres.2005.08.007

9. Grasley Z, Lange D (2007) Constitutive modeling of the aging viscoelastic properties of portland cement paste. Mech Time-Depend Mater 11:175-198. https://doi.org/10. 1007/s11043-007-9043-4

10. Poole R (2012) The Deborah and Weissenberg numbers. Br. Soc. Rheol-Rheol Bull 53:32-39

11. Mohan MK, Rahul AV, De Schutter G, Van Tittelboom K (2021) Extrusion-based concrete 3D printing from a material perspective: a state-of-the-art review. Cem Concr Compos 115:103855. https://doi.org/10.1016/j. cemconcomp.2020.103855

12. Maia L, Figueiras J (2012) Early-age creep deformation of a high strength self-compacting concrete. Constr Build Mater 34:602-610. https://doi.org/10.1016/j.conbuildmat.2012. 02.083

13. Leemann A, Lura P, Loser R (2011) Shrinkage and creep of SCC - The influence of paste volume and binder composition. Constr Build Mater 25:2283-2289. https://doi.org/10. 1016/j.conbuildmat.2010.11.019

14. Rozière E, Granger S, Turcry Ph, Loukili A (2007) Influence of paste volume on shrinkage cracking and fracture properties of self-compacting concrete. Cement Concr Compos 29:626-636. https://doi.org/10.1016/j. cemconcomp.2007.03.010

15. Briffaut M, Benboudjema F, Torrenti J-M, Nahas G (2012) Concrete early age basic creep: experiments and test of rheological modelling approaches. Constr Build Mater 36:373-380. https://doi.org/10.1016/j.conbuildmat.2012. 04.101

16. Han B, Xie H-B, Zhu L, Jiang P (2017) Nonlinear model for early age creep of concrete under compression strains. Constr Build Mater 147:203-211. https://doi.org/10.1016/j. conbuildmat.2017.04.119

17. De Schutter G (1999) Degree of hydration based Kelvin model for the basic creep of early age concrete. Mater Struct 32:260-265. https://doi.org/10.1007/BF02479595

18. Niyogi AK, Hsu P, Meyers BL (1973) The influence of age at time of loading on basic and drying creep. Cem Concr Res 3:633-644. https://doi.org/10.1016/0008-8846(73)90100-2

19. Østergaard L, Lange DA, Altoubat SA, Stang H (2001) Tensile basic creep of early-age concrete under constant load. Cem Concr Res 31:1895-1899. https://doi.org/10. 1016/S0008-8846(01)00691-3
20. Ghosh RS (1972) Creep of portland cement paste at early ages. Mat Constr 5:93-97. https://doi.org/10.1007/ BF02478438

21. Bos F, Wolfs R, Ahmed Z, Salet T (2016) Additive manufacturing of concrete in construction: potentials and challenges of 3D concrete printing. Virtual Phys Prototyp 11:1-17. https://doi.org/10.1080/17452759.2016.1209867

22. Bazant ZP, Chern JC (1985) Concrete creep at variable humidity: constitutive law and mechanism. Mater Struct 18:1. https://doi.org/10.1007/BF02473360

23. Burgers JM (1935) Mechanical considerations-model systems-phenomenological theories of relaxation and of viscosity. In First Report on Viscosity and Plasticity; Nordemann Publishing: New York, NY, USA, 1935; Chapter1, pp. 5-67

24. Casagrande L, Esposito L, Menna C et al (2020) Effect of testing procedures on buildability properties of 3D-printable concrete. Constr Build Mater 245:118286. https://doi. org/10.1016/j.conbuildmat.2020.118286

25. Tschoegl NW (1989) Energy storage and dissipation in a linear viscoelastic material. In: The phenomenological theory of linear viscoelastic behavior. Springer Berlin, Heidelberg. https://doi.org/10.1007/978-3-642-73602-5_9

26. Banks HT, Hu S, Kenz ZR (2011) A brief review of elasticity and viscoelasticity for solids. Adv Appl Math Mech 3:1-51. https://doi.org/10.4208/aamm.10-m1030

27. Asprone D, Auricchio F, Menna C, Mercuri V (2018) 3D printing of reinforced concrete elements: technology and design approach. Constr Build Mater 165:218-231. https:// doi.org/10.1016/j.conbuildmat.2018.01.018

28. EN 12390-3: 2019 - Testing hardened concrete - Part 3: Compressive strength of test specimens. In: iTeh Standards Store. https://standards.iteh.ai/catalog/standards/cen/ 7eb738ef-44af-436c-ab8e-e6561571302c/en-12390-32019. Accessed 21 May 2021

29. ASTM D 2166 (2000) Standard test method for unconfined compressive strength of cohesive soil, Annual book of ASTM Standards, American Society for Testing and Materials, Philadelphia 4 (08) (2003)

30. Wolfs RJM, Bos FP, Salet TAM (2018) Early age mechanical behaviour of 3D printed concrete: numerical modelling and experimental testing. Cem Concr Res 106:103-116. https://doi.org/10.1016/j.cemconres.2018.02. 001

31. Wolfs RJM, Bos FP, Salet TAM (2019) Triaxial compression testing on early age concrete for numerical analysis of 3D concrete printing. Cement Concr Compos 104:103344. https://doi.org/10.1016/j.cemconcomp.2019.103344

32. Yu K, McGee W, Ng TY et al (2021) 3D-printable engineered cementitious composites (3DP-ECC): Fresh and hardened properties. Cem Concr Res 143:106388. https:// doi.org/10.1016/j.cemconres.2021.106388

33. Bažant ZP (2001) Prediction of concrete creep and shrinkage: past, present and future. Nucl Eng Des 203:27-38. https://doi.org/10.1016/S0029-5493(00)00299-5

34. Bažant Z (1995) Creep and shrinkage prediction model for analysis and design of concrete structures-model B3. Mater Struct 28:357-365. https://doi.org/10.1007/ BF02473152

35. Panda B, Lim JH, Tan MJ (2019) Mechanical properties and deformation behaviour of early age concrete in the context 
of digital construction. Compos B Eng 165:563-571. https://doi.org/10.1016/j.compositesb.2019.02.040

36. Paul S, van Zijl G, Tan MJ, Gibson I (2018) A review of 3D concrete printing systems and materials properties: current status and future research prospects. Rapid Prototyp J 24:00-00. https://doi.org/10.1108/RPJ-09-2016-0154

37. Tay YWD, Ting GH, Qian Y et al (2018) Time gap effect on bond strength of 3D-printed concrete. Virtual Phys Prototyp 14:104-113. https://doi.org/10.1080/17452759.2018. 1500420
38. Le T, Austin S, Lim S et al (2012) Hardened properties of high-performance printing concrete. Cem Concr Res 42:558-566. https://doi.org/10.1016/j.cemconres.2011.12. 003

Publisher's Note Springer Nature remains neutral with regard to jurisdictional claims in published maps and institutional affiliations. 